\title{
DIRICHLET-FINITE ANALYTIC AND HARMONIC FUNCTIONS ARE BMO
}

\author{
J. L. SCHIFF
}

(Communicated by Paul S. Muhly)

\begin{abstract}
Based on a result of F. John, an elementary proof is given of the fact that Dirichlet-finite analytic and Dirichlet-finite harmonic functions are of bounded mean oscillation in the unit disk.
\end{abstract}

1. In [5] Metzger proved the rather surprising result that the space of Dirichletfinite analytic functions on a hyperbolic Riemann surface belong to the space BMO. Subsequently, in [4], Kusunoki and Taniguchi found that the same result holds for Dirichlet-finite harmonic functions on Riemann surfaces of finite type. In this note we give an elementary proof, based on a result of John [2], that in the unit disk Dirichlet-finite analytic and harmonic functions are BMO.

2. Denote the unit disk by $U:|z|<1$, and

$$
\begin{gathered}
A D(U)=\left\{f \in A(U): D_{U}(f)=\int_{U} \int\left|f^{\prime}(z)\right|^{2} d x d y<\infty\right\}, \\
H D(U)=\left\{u \in H(U): D_{U}(u)=\int_{U} \int|\operatorname{grad} u|^{2} d x d y<\infty\right\},
\end{gathered}
$$

as the spaces of Dirichlet-finite analytic, and Dirichlet-finite harmonic, functions, respectively.

Let $\operatorname{BMO} A(U)$ be the space of analytic functions on $U$ which belong to the Hardy class $H^{2}(U)$ and satisfy

$$
\sup _{\zeta \in U} \int_{U} \int\left|f^{\prime}(z)\right|^{2} \log \left|\frac{1-\bar{\zeta} z}{z-\zeta}\right| d x d y<\infty,
$$

and define $\mathrm{BMO} H(U)$ analogously for harmonic functions, replacing $\left|f^{\prime}\right|^{2}$ by $|\operatorname{grad} u|^{2}$.

A sufficient condition for a real-valued differentiable function $u$ to belong to $\operatorname{BMO}(U)$ in the sense of John-Nirenberg [3] has been given by John [2], namely: $\sup _{z \in U}(1-|z|)|\operatorname{grad} u(z)|<\infty$.

Received by the editors July 19, 1988.

1980 Mathematics Subject Classification (1985 Revision). Primary 30D99; Second 30D55. 
We can now readily establish the following:

Theorem. $A D(U) \subseteq \mathrm{BMO} A(U)$.

Proof. Firstly, $A D(U) \subseteq H^{2}(U)$ (cf. Heins [1]). Take $f \in A D(U), f=$ $u+i v$. By the areal mean value property applied to $\operatorname{Re}\left[\left(f^{\prime}\right)^{2}\right], \operatorname{Im}\left[\left(f^{\prime}\right)^{2}\right]$,

$$
\left(f^{\prime}(z)\right)^{2}=\frac{1}{\pi \rho^{2}} \int_{0}^{\rho} \int_{0}^{2 \pi}\left[f^{\prime}\left(z+r e^{i \theta}\right)\right]^{2} r d r d \theta
$$

where $\rho=1-|z|$. Then

$$
|\operatorname{grad} u(z)|^{2}=\left|f^{\prime}(z)\right|^{2} \leq \frac{1}{\pi(1-|z|)^{2}} \int_{U} \int\left|f^{\prime}(z)\right|^{2} d x d y=\frac{1}{\pi(1-|z|)^{2}} D_{U}(f) .
$$

Therefore,

$$
\sup _{z \in U}(1-|z|)|\operatorname{grad} u(z)| \leq \sqrt{\frac{1}{\pi} D_{U}(f)}<\infty .
$$

Since $u \in h^{2}(U)$, the John result implies $u \in \operatorname{BMOH}(U)$, and hence $f \in$ $\mathrm{BMO} A(U)$.

For any function $u \in H D(U), u \in \operatorname{Re} f$ for some $f \in A D(U)$, and the preceding proof yields:

Corollary. $H D(U) \subseteq \mathrm{BMO} H(U)$.

\section{REFERENCES}

1. M. Heins, Hardy classes on Riemann surfaces, Lecture Notes in Math., 98, Springer-Verlag, 1969.

2. F. John, Functions whose gradients are bounded by the reciprocal distance from the boundary of their domain, Russian Math. Surveys 29 (1974), 170-175.

3. F. John and L. Nirenberg, On functions of bounded mean oscillation, Comm. Pure Appl. Math. 14 (1961), 415-426.

4. Y. Kusunoki and M. Taniguchi, Remarks on functions of bounded mean oscillation on Riemann surfaces, Kodai Math. J. 6 (1983), 434-442.

5. T. Metzger, On BMOA for Riemann surfaces, Canad. Math. J. 33 (1981), 1255-1260.

Department of Mathematics, University of Auckland, Auckland, New Zealand

Current address: Department of Mathematics, University of York, Heslington, York Y01 5DD England 